\title{
Sleep apnea and risk of traumatic brain injury and associated mortality and healthcare costs: a population-based cohort study
}

\author{
Shih-Yi Lin ${ }^{1,2}$, Weishan Chen ${ }^{3,4}$, Tomor Harnod ${ }^{5,6}$, Cheng-Li Lin ${ }^{3,4}$, Wu-Huei Hsu ${ }^{1,7}$, Cheng-Chieh Lin ${ }^{1,8}$, \\ Yun-Lun Chang ${ }^{1,2}$, I-Kuan Wang ${ }^{1,2}$, Chia-Hung Kao ${ }^{1,9,10,11}$ \\ ${ }^{1}$ Graduate Institute of Biomedical Sciences, College of Medicine, China Medical University, Taichung; ${ }^{2}$ Division of Nephrology and Kidney \\ Institute, China Medical University Hospital, Taichung; ${ }^{3}$ Management Office for Health Data, China Medical University Hospital, Taichung; \\ ${ }^{4}$ College of Medicine, China Medical University, Taichung; ${ }^{5}$ Department of Neurosurgery, Hualien Tzu Chi General Hospital, Buddhist Tzu Chi \\ Medical Foundation, Hualien; ${ }^{6}$ College of Medicine, Tzu Chi University, Hualien; ${ }^{7}$ Division of Pulmonary and Critical Care Medicine, China \\ Medical University Hospital, China Medical University, Taichung; ${ }^{8}$ Department of Family Medicine, ${ }^{9}$ Department of Nuclear Medicine and PET \\ Center, Center of Augmented Intelligence in Healthcare, China Medical University Hospital, Taichung; ${ }^{10}$ Department of Bioinformatics and Medical \\ Engineering, Asia University, Taichung; ${ }^{11}$ Center of Augmented Intelligence in Healthcare, China Medical University Hospital, Taichung \\ Contributions: (I) Conception and design: SY Lin, CH Kao; (II) Administrative support: None; (III) Provision of study materials or patients: CH Kao; \\ (IV) Collection and assembly of data: SY Lin, WS Chen, CH Kao; (V) Data analysis and interpretation: All authors; (VI) Manuscript writing: All \\ authors; (VII) Final approval of manuscript: All authors. \\ Correspondence to: Chia-Hung Kao, MD. Graduate Institute of Biomedical Sciences and School of Medicine, College of Medicine, China Medical \\ University, No. 2, Yuh-Der Road, Taichung 40447. Email: d10040@mail.cmuh.org.tw; dr.kaochiahung@gmail.com.
}

Background: The objective of this study was aimed to investigate whether sleep apnea patients had a higher risk of traumatic brain injury.

Methods: Data were collected from the Taiwan Longitudinal Health Insurance Database during the period of 2000-2012. The study cohort comprised 6,456 patients aged $\geq 20$ years with a first diagnosis of sleep apnea. The primary outcome was the incidence of traumatic brain injury. Kaplan-Meier survival analysis and Cox proportional-hazards modeling were used.

Results: After adjustments for associated comorbidities and hypnotic medications, sleep apnea patients were associated with a 1.19-fold higher risk of traumatic brain injury (95\% CI, 1.07-1.33) compared with patients without sleep apnea. Sleep apnea patients who took benzodiazepine (BZD) had a 1.30-fold increased risk of traumatic brain injury compared with patients without sleep apnea (95\% CI, 1.14-1.49). However, this risk was not statistically significant, with a 1.03 -fold risk of traumatic brain injury in sleep apnea patients without BZD use (95\% CI, 0.84-1.25) compared with patients without sleep apnea. Compared with patients without sleep apnea, the risk of traumatic brain injury in sleep apnea patients aged 65-79 years old was higher (adjusted hazard ratio, 1.36; 95\% CI, 1.06-1.74).

Conclusions: Sleep apnea patients, regardless of hypnotic use, had a higher risk of traumatic brain injury compared with patients without sleep apnea.

Keywords: Sleep apnea; traumatic brain injury; hypnotic

Submitted Aug 12, 2019. Accepted for publication Oct 10, 2019.

doi: $10.21037 /$ atm.2019.10.88

View this article at: http://dx.doi.org/10.21037/atm.2019.10.88 


\section{Introduction}

Sleep apnea, a condition characterized by periods of paused or shallow breathing during sleep, affects a large number of the world's population. Previous research has established that sleep disturbances are associated with an increased risk of acute coronary syndrome (1), stroke (2), neurodegenerative disease (3), type 2 diabetes (4), and obesity (5). The proposed mechanisms underlying this elevated risk include increased oxidative stress (6), brain hypoperfusion (7), endothelial dysfunction (8), chronic elevation of glucocorticoids (9), and elevated plasma levels of homocysteine (10).

However, few studies have investigated the impacts of sleep apnea itself on accidental injuries. Sleep disorders have been linked with daytime impaired attention and even attention-deficit/hyperactivity disorder in adults $(11,12)$. However, until now, whether sleep apnea patients experience more hazardous crucial injuries, such as traumatic brain injuries, lacks scientific evidence. Additionally, whether using continuous positive airway pressure (CPAP), the common treatment strategy for sleep apnea, could help alleviate the risk of brain injuries remains unknown. Therefore, we conducted a large nationwide controlled cohort study in Taiwan to investigate the possible risks of traumatic brain injuries in sleep apnea patients.

\section{Methods}

\section{Data source}

The National Health Insurance program was established in 1995, and it covers more than $99 \%$ of Taiwan's population (13). For this retrospective cohort study, we used a subdatabase of the National Health Insurance Research Database (NHIRD) called the Longitudinal Health Insurance Database 2000 (LHID2000). The LHID2000 contains data of 1 million randomly selected patients from the NHIRD.

\section{Ethics statement}

The NHIRD encrypts patient personal information to protect privacy and provides researchers with anonymous identification numbers associated with relevant claims information, including sex, date of birth, medical services received, and prescriptions. Therefore, patient consent is not required to access the NHIRD. This study was approved as fulfilling the conditions for exemption by the Institutional Review Board (IRB) of China Medical University (CMUH104-REC2-115-CR4). The IRB also specifically waived the consent requirement.

\section{Sampled participants}

In this study, patients older than 20 years and with newly diagnosed sleep apnea (ICD-9-CM 327.2, 780.51, 780.53, and 780.57) between 2000 and 2012 in LHID2000 were enrolled. The diagnosis of sleep apnea was defined as two outpatient visits or one inpatient visit for sleep apnea. The coding of sleep apnea in the NHIRD has been used for several investigations, including many previous successfully published publications. Therefore, the coding of sleep apnea could be considered reliable and accurate (14-16). The index date was the date of first sleep apnea diagnosis. We excluded patients with missing information regarding urbanization level or occupation, who had a history of traumatic brain injury (ICD-9-CM 310.2, 800, 801, 803, $804,850-854,959.01$ ), or who withdrew from the insurance program before the index date. The control cohort was selected from the patients who had never been diagnosed with sleep apnea and the index date was a randomly assigned date between 2000 and 2012. The exclusion criteria were the same as in the sleep apnea cohort. Then, the cohort without sleep apnea was 4-fold size matched with the sleep disorder cohort by index year, age (every 5 years), and gender. The selection process is shown in Figure 1.

\section{Outcome and relevant variables}

The outcome discussed in this study was traumatic brain injury (ICD-9-CM 310.2, 800, 801, 803, 804, 850-854, and 959.01). The end of the study was defined as occurrence of a traumatic brain injury event, withdrawal from the insurance program, or the end of 2013. We considered patients' urbanization level and occupation. Urbanization was divided into four levels according to the population density of the residential area, with Level 1 as the most urbanized and Level 4 as the least. The occupations were white collar, blue collar, and other. Patients belonged to the "other" occupation if they were primarily retired, unemployed, belonged to another low-income population. To clarify the association between sleep apnea and brain injuries, we attempted to adjust and control for medical conditions that tend to cause falls in patients (i.e., musculoskeletal disease, including osteoporosis, fracture, and spinal cord injuries; rheumatological disease; internal diseases that cause 


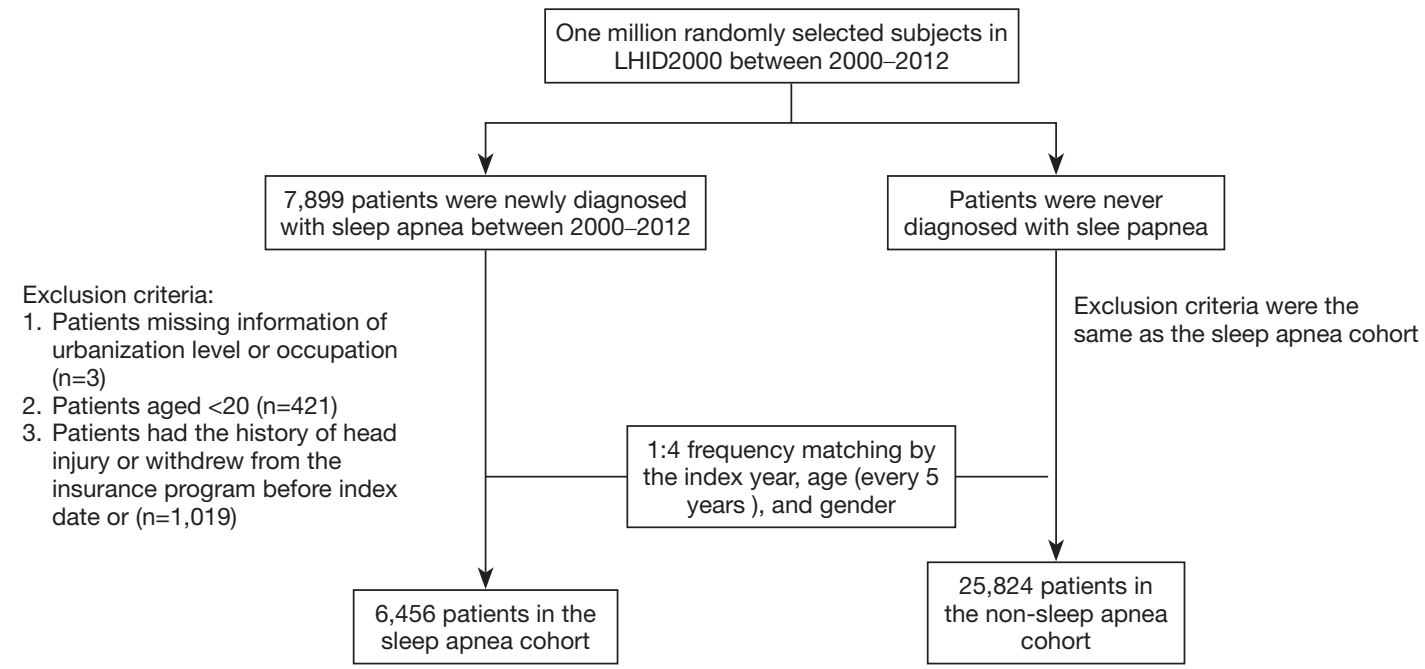

Figure 1 Flowchart of patient selection.

fragility, including liver disease, renal disease, and factors of metabolic syndrome; common eye disorders, including cataract, presbyopia, myopia, and macular degeneration; and some sedative use). The comorbidities were defined as a diagnosis prior to the endpoint and included diabetes (ICD-9-CM 250 and A181), hypertension (ICD-9-CM 401-405, A260, and A269), hyperlipidemia (ICD-9-CM 272 and A182), spinal cord injury (ICD-9-CM 806 and 952), osteoporosis (ICD-9-CM 733.0), fracture (ICD-9CM 800-829 and A47X), alcohol-related disease (ICD-9CM 291, 303, 305.00, 305.01, 305.02, 305.03, 790.3, V11.3, and A215), cirrhosis (ICD-9-CM 571 and A347), cataract (ICD-9-CM 366 and A231), presbyopia or myopia (ICD-9CM 360.21, 367.1, and 367.4), macular degeneration (ICD9-CM 362.5), stroke (ICD-9-CM 430-438), chronic kidney disease (CKD) or end-stage renal diseases (ESRD) (ICD-9CM 580-589 and A350), dementia (ICD-9-CM 290, 294.1, and 331.0), and rheumatoid arthritis (ICD-9-CM 714.0). We also considered patients who received benzodiazepines (BZDs), nonbenzodiazepines (non-BZDs), or antipsychotic medications (17-19) and those who received CPAP treatment (ICD-9-OP 93.90) during the study period.

In addition to evaluating the association between sleep apnea and occurrence of brain injuries, we evaluated the association between sleep apnea and severities of brain injuries. Information regarding Glasgow Coma Scale or IMPACT score (20) was unavailable in the NHIRD. Therefore, we used intensive care unit (ICU) use and mortality following a brain injury episode as our research outcomes.

\section{Data availability statement}

The dataset used in this study is held by the Taiwan Ministry of Health and Welfare (MOHW). The MOHW must approve a researcher's application to access this data. Any researcher interested in accessing this dataset can submit an application form to the $\mathrm{MOHW}$ requesting access. Please contact the staff of MOHW (Email: stcarolwu@mohw.gov.tw) for further assistance. Taiwan Ministry of Health and Welfare Address: No.488, Sec. 6, Zhongxiao E. Rd., Nangang Dist., Taipei City 115, Taiwan (R.O.C.). Phone: $+886-2-8590-6848$. All relevant data are within the paper.

\section{Statistical analysis}

Table 1 shows the demographics of patients in sleep apnea cohort and the cohort without sleep apnea. To test the differences in these factors between two groups, the continuous variable was tested using a $t$ test and categorical variables were testing using a Chi-squared test. We estimated the incidence rate and hazard ratio of traumatic brain injury for patients with sleep apnea compared with controls in Table 2. The incidence rate was estimated by the number of events that occurred and person-years. Univariable and multivariable Cox proportional-hazard regression models estimated the hazard ratio (HR) and $95 \%$ confidence interval (CI) of traumatic brain injury between the two groups. Variables found to be statistically significant in the univariable model were further included 
Page 4 of 12

Lin et al. Sleep apnea and risk of brain injury

Table 1 Demographic characteristics and comorbidities in cohorts with and without sleep apnea

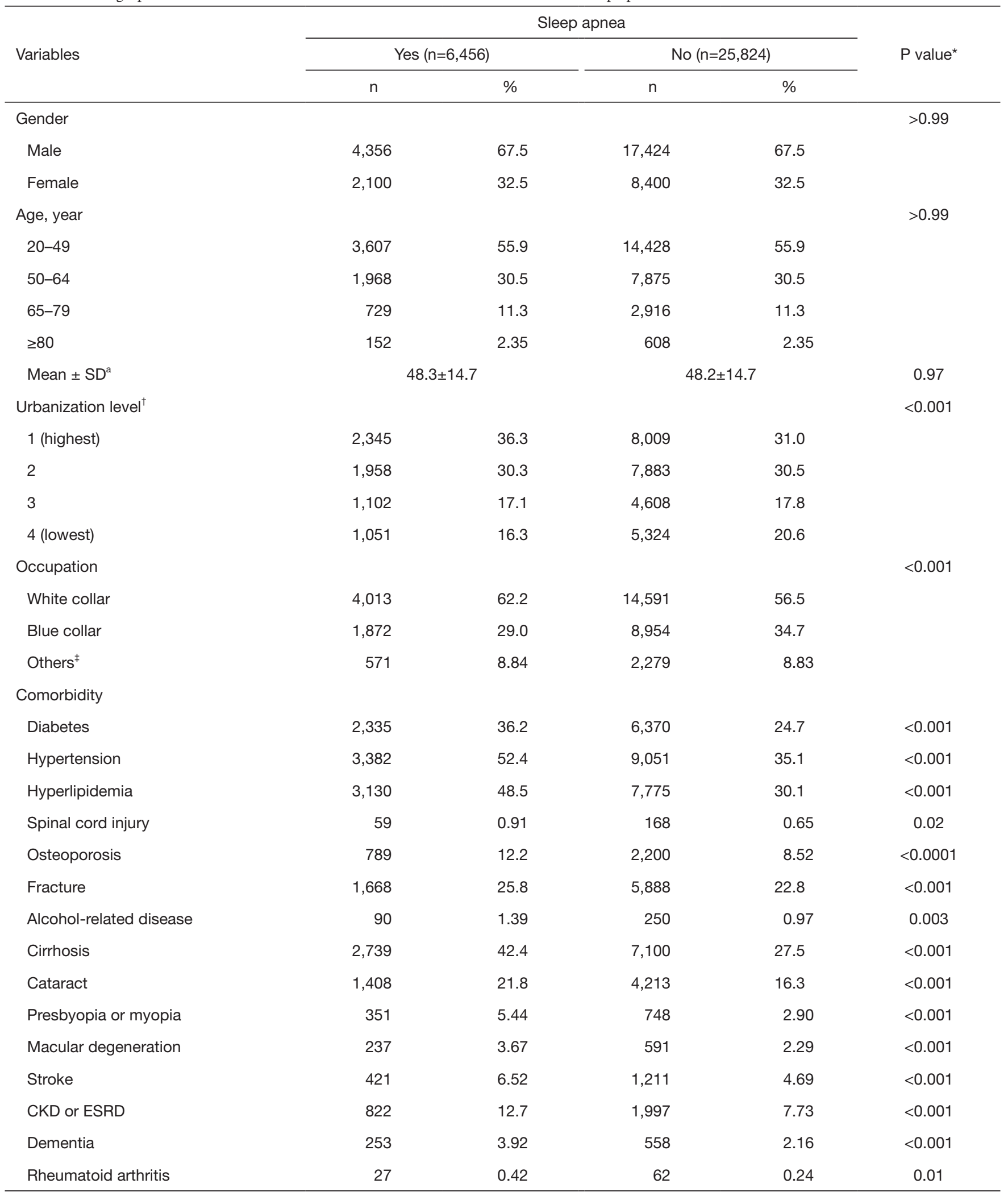

Table 1 (Continued) 
Table 1 (Continued)

\begin{tabular}{|c|c|c|c|c|c|}
\hline \multirow{2}{*}{ Variables } & \multicolumn{4}{|c|}{ Sleep apnea } & \multirow{2}{*}{$P$ value* } \\
\hline & $\mathrm{n}$ & $\%$ & $\mathrm{n}$ & $\%$ & \\
\hline \multicolumn{6}{|l|}{ Medication } \\
\hline Benzodiazepine & 4,291 & 66.5 & 11,027 & 42.7 & $<0.001$ \\
\hline Anti-psychotic & 1,121 & 17.4 & 2,624 & 10.2 & $<0.001$ \\
\hline
\end{tabular}

Table 2 Incidence and hazard ratio of traumatic brain injury for patients with sleep apnea compared with controls stratified by gender, age, comorbidity, and medication

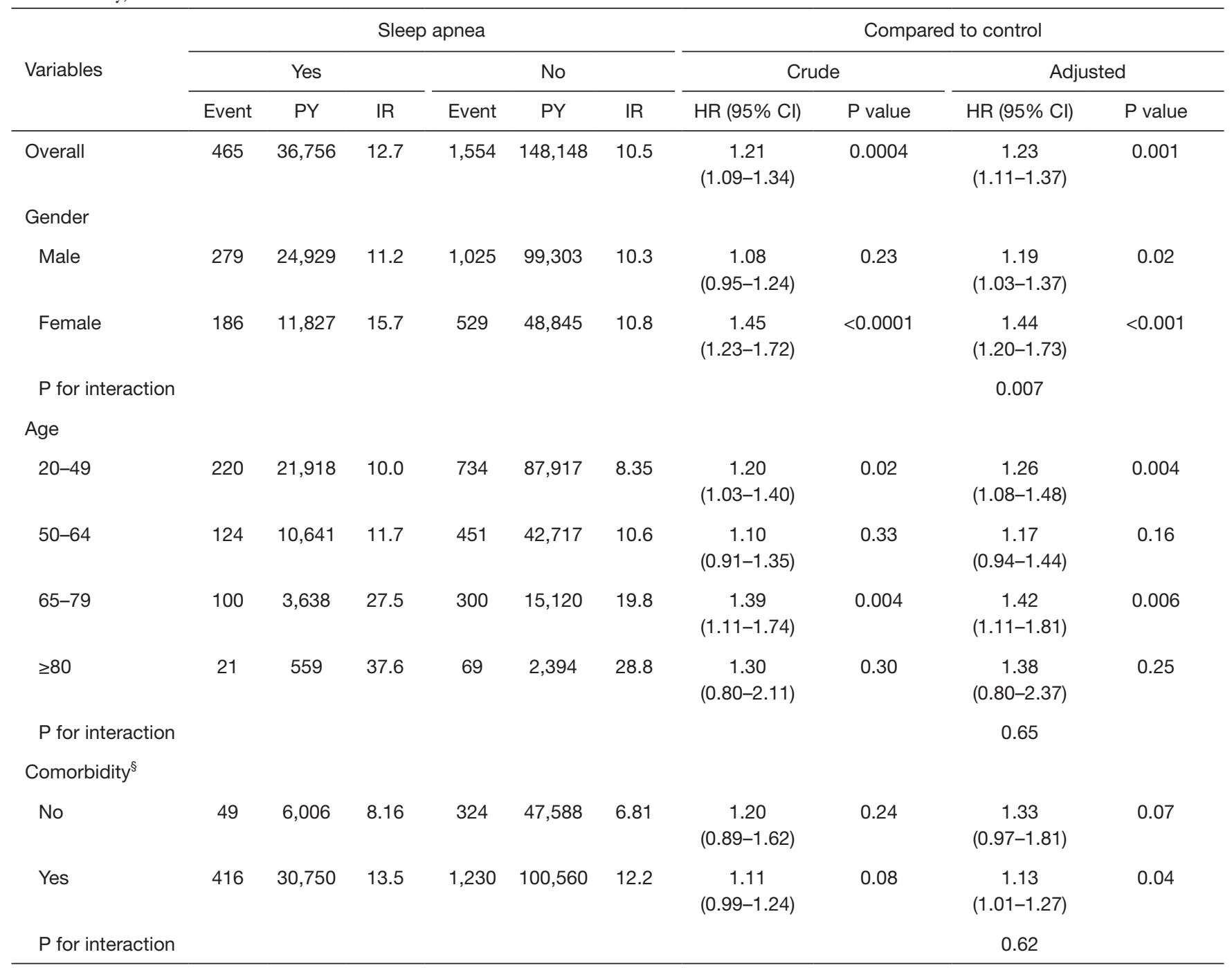

Table 2 (Continued) 
Table 2 (Continued)

\begin{tabular}{|c|c|c|c|c|c|c|c|c|c|c|}
\hline \multirow{2}{*}{ Variables } & \multicolumn{6}{|c|}{ Sleep apnea } & \multicolumn{4}{|c|}{ Compared to Control } \\
\hline & Event & PY & IR & Event & PY & IR & $\mathrm{HR}(95 \% \mathrm{Cl})$ & $P$ value & $\mathrm{HR}(95 \% \mathrm{Cl})$ & $P$ value \\
\hline \multicolumn{11}{|l|}{ Medication } \\
\hline \multicolumn{11}{|l|}{ Benzodiazepine } \\
\hline Yes & 344 & 26,165 & 13.1 & 718 & 73,613 & 9.75 & $\begin{array}{c}1.36 \\
(1.20-1.55)\end{array}$ & $<0.0001$ & $\begin{array}{c}1.31 \\
(1.14-1.49)\end{array}$ & $<0.001$ \\
\hline$P$ for interaction & & & & & & & & & 0.02 & \\
\hline \multicolumn{11}{|l|}{ Non-BZD } \\
\hline Yes & 224 & 15,013 & 14.9 & 314 & 25,230 & 12.4 & $\begin{array}{c}1.23 \\
(1.03-1.46)\end{array}$ & 0.02 & $\begin{array}{c}1.23 \\
(1.03-1.46)\end{array}$ & 0.02 \\
\hline$P$ for interaction & & & & & & & & & 0.45 & \\
\hline \multicolumn{11}{|l|}{ Anti-psychotic } \\
\hline No & 366 & 29,645 & 12.3 & 1,332 & 130,188 & 10.2 & $\begin{array}{c}1.21 \\
(1.07-1.35)\end{array}$ & 0.002 & $\begin{array}{c}1.23 \\
(1.09-1.39)\end{array}$ & 0.001 \\
\hline Yes & 99 & 7,111 & 13.9 & 222 & 17,960 & 12.4 & $\begin{array}{c}1.14 \\
(0.90-1.45)\end{array}$ & 0.28 & $\begin{array}{c}1.05 \\
(0.82-1.35)\end{array}$ & 0.70 \\
\hline$P$ for interaction & & & & & & & & & 0.60 & \\
\hline
\end{tabular}

PY, person-years; IR, incidence rate, per 1,000 person-years; HR, hazard ratio; Cl, confidence interval. Variables found to be statistically significant in the univariable model were further included in the multivariable model. Adjusted for sex, age, diabetes, hypertension, spinal cord injury, osteoporosis, fracture, alcohol-related disease, cirrhosis, cataract, macular degeneration, stroke, CKD or ESRD, dementia, and benzodiazepine, non-benzodiazepine, and antipsychotic medication use. Comorbidity : patients with any one of the comorbidities were classified into the comorbidity group.

in the multivariable model. To address the concern of constant proportionality, we examined the proportionalhazard model assumption using a test of scaled Schoenfeld residuals. Results revealed no significant relationship between Schoenfeld residuals for sleep apnea and followup time $(\mathrm{P}=0.70)$ in the model evaluating traumatic brain injury risk. We also used the Kaplan-Meier survival curve to describe the cumulative incidence of traumatic brain injury in the two groups and the log-rank test to test the differences between the two groups. Table 3 presents the odds ratios of ICU utilization in hospitalized patients for traumatic brain injury between the two groups and estimated using univariable and multivariable unconditional logistic regression. We estimated the odds ratio of mortality for traumatic brain injury patients between the two groups
(Table 4). Data were analyzed using SAS statistical software (Version 9.4 for Windows; SAS Institute, Inc., Cary, NC, USA). The statistical significance of this study was a twotailed $\mathrm{P}$ value of $<0.05$.

\section{Results}

This study included 6,456 sleep apnea patients and 25,824 patients without sleep apnea; no differences in gender and age were observed between the two groups. The mean follow-up rate was more than $93 \%$ in both cohorts. The sleep apnea cohort had a higher percentage of patients living in the most urbanized area and a higher percentage of patient occupations were white collar occupations. The sleep apnea cohort had higher proportions of comorbidities 
Table 3 Odds ratio of ICU in hospitalized patients with traumatic brain injury between two groups stratified by gender, age, and comorbidity

\begin{tabular}{|c|c|c|c|c|c|c|}
\hline \multirow{3}{*}{ Variables } & \multicolumn{2}{|l|}{ ICU } & \multicolumn{4}{|c|}{ Compared to control } \\
\hline & \multirow{2}{*}{$\begin{array}{l}\text { In sleep apnea group } \\
\qquad(\mathrm{N}=85)\end{array}$} & \multirow{2}{*}{$\begin{array}{l}\text { In control group } \\
\qquad(\mathrm{N}=354)\end{array}$} & \multicolumn{2}{|c|}{ Crude } & \multicolumn{2}{|c|}{ Adjusted } \\
\hline & & & OR $(95 \% \mathrm{Cl})$ & $P$ value & $(95 \% \mathrm{Cl})$ & $P$ value \\
\hline Overall & $29(34.1)$ & $121(34.2)$ & $1.00(0.61-1.64)$ & 0.99 & $0.92(0.54-1.57)$ & 0.76 \\
\hline \multicolumn{7}{|l|}{ Gender } \\
\hline Female & 8 (29.6) & $19(21.8)$ & $1.51(0.57-3.98)$ & 0.41 & $1.35(0.47-3.87)$ & 0.58 \\
\hline \multicolumn{7}{|l|}{ Age } \\
\hline $20-49$ & $8(23.5)$ & $40(29.2)$ & $0.75(0.31-1.79)$ & 0.51 & $0.57(0.21-1.54)$ & 0.27 \\
\hline $50-64$ & $9(36.0)$ & $44(38.6)$ & $0.89(0.36-2.2)$ & 0.81 & $0.82(0.31-2.17)$ & 0.69 \\
\hline \multicolumn{7}{|c|}{ Comorbidity ${ }^{\S}$} \\
\hline No & $0(0.00)$ & 5 (14.3) & - & - & - & - \\
\hline Yes & $29(35.4)$ & $116(36.4)$ & $0.96(0.58-1.59)$ & 0.87 & 1.01 (0.60-1.69) & 0.97 \\
\hline
\end{tabular}

OR, odds ratio; $\mathrm{Cl}$, confidence interval. Variables found to be statistically significant in the univariable model were further included in the multivariable model. Adjusted for sex, age, diabetes, and fracture. Comorbidity $\$$ : patients with any one of the comorbidities were classified as the comorbidity group.

Table 4 Odds ratio of mortality for traumatic brain injury patients between two groups stratified by gender, age, and comorbidity

\begin{tabular}{|c|c|c|c|c|c|c|}
\hline \multirow{2}{*}{ Variables } & \multicolumn{2}{|c|}{ Mortality } & \multicolumn{4}{|c|}{ Compared to control } \\
\hline & $\begin{array}{l}\text { In sleep apnea group } \\
\qquad(\mathrm{N}=465)\end{array}$ & $\begin{array}{l}\text { In control group } \\
\qquad(\mathrm{N}=1,554)\end{array}$ & OR $(95 \% \mathrm{Cl})$ & $P$ value & $(95 \% \mathrm{Cl})$ & $P$ value \\
\hline Overall & $6(1.29)$ & $10(0.64)$ & $2.02(0.73-5.58)$ & 0.18 & $2.56(0.89-7.41)$ & 0.08 \\
\hline \multicolumn{7}{|l|}{ Gender } \\
\hline Female & $2(1.08)$ & $2(0.38)$ & $2.86(0.4-20.48)$ & 0.29 & $3.36(0.42-26.5)$ & 0.25 \\
\hline \multicolumn{7}{|l|}{ Age } \\
\hline $20-49$ & $1(0.45)$ & $2(0.27)$ & $1.67(0.15-18.52)$ & 0.68 & $2.18(0.19-24.9)$ & 0.53 \\
\hline $50-64$ & $1(0.81)$ & $3(0.67)$ & $1.21(0.13-11.77)$ & 0.87 & $1.28(0.12-13.8)$ & 0.84 \\
\hline \multicolumn{7}{|c|}{ Comorbidity ${ }^{\S}$} \\
\hline No & $0(0)$ & $3(0.52)$ & - & & - & \\
\hline Yes & $6(1.60)$ & $7(0.71)$ & $2.44(0.86-6.92)$ & 0.09 & $2.43(0.84-7.03)$ & 0.10 \\
\hline
\end{tabular}

OR, odds ratio; $\mathrm{Cl}$, confidence interval. Variables found to be statistically significant in the univariable model were further included in the multivariable model. Adjusted for age and CKD or ESRD. Comorbiditys: patients with any one of the comorbidities were classified as the comorbidity group. 


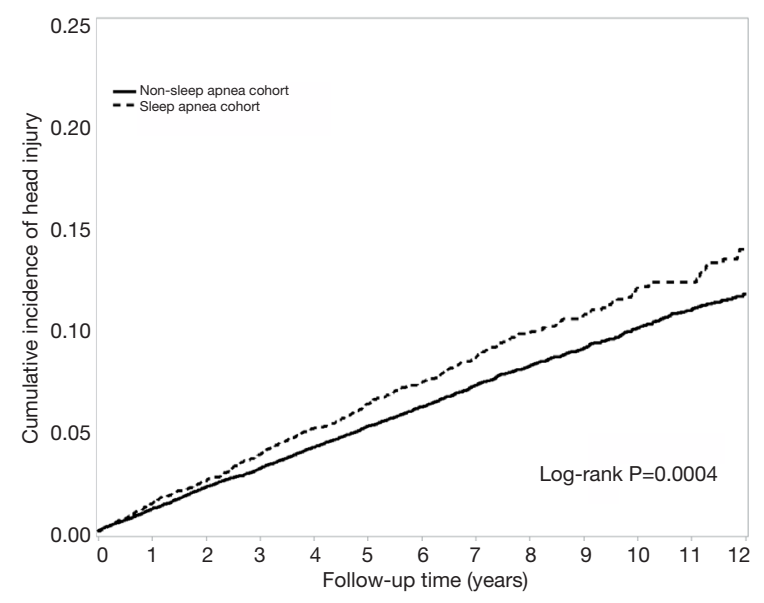

Figure 2 Cumulative incidence of traumatic brain injury in the sleep disorder and non-sleep disorder cohorts.

and medications used than the cohort without sleep apnea.

The incidence rate of traumatic brain injury for cohorts with and without sleep apnea was 12.7 and 10.5 per 1,000 person-years, respectively. Sleep apnea patients were 1.23fold more likely to develop traumatic brain injury (95\% CI, 1.11-1.37) than the control cohort after adjustment for sex, age, diabetes, hypertension, spinal cord injury, osteoporosis, fracture, alcohol-related disease, cirrhosis, cataract, macular degeneration, stroke, CKD or ESRD, dementia, and BZD, non-BZD, and antipsychotic medication use. A KaplanMeier survival curve also showed that the sleep apnea cohort had a higher incidence of traumatic brain injury than the cohort without sleep apnea (Figure 2). We then stratified patients by gender, age, comorbidities, and medications. Patients were placed in the comorbidity group if they had any one of the identified comorbidities. Sleep apnea patients exhibited an increased risk of traumatic brain injury (adjusted HR 1.44, 95\% CI, 1.20-1.73) among women, and a difference in men between the two groups was noted (adjusted HR 1.19, 95\% CI, 1.03-1.37). For age stratifications, we only found groups of patients aged 20-79 and 65-79 where sleep apnea patients had higher risks of traumatic brain injury than patients without sleep apnea (adjusted HR 1.26, 95\% CI, 1.08-1.48: adjusted HR 1.42, 95\% CI, 1.11-1.81). For patients in the comorbidity group, the sleep apnea cohort had a 1.13 -fold risk of traumatic brain injury $(95 \%$ CI, 1.01-1.27). We then considered medication used and found that that patients with sleep apnea had a higher risk of traumatic brain injury than patients without sleep apnea in some stratifications (BZD users: adjusted HR 1.31, 95\% CI, 1.14-1.49; non-BZD nonusers: adjusted HR 1.17, 95\% CI,
1.02-1.35; antipsychotic nonuser: adjusted HR 1.23, 95\% CI, $1.09-1.39)$.

We then explored the risk of ICU utilization in hospitalized patients with traumatic brain injury between the two groups (Table 3) and found that no statistically significant differences. After adjusting for age, sex, diabetes, and fractures, we determined that the odds ratio (OR) of traumatic brain was not significant for sleep apnea patients with ICU use compared with patients without sleep apnea who utilized the ICU (adjusted OR 0.92, 95\% CI, 0.541.57). We further discussed the risk of death caused by traumatic brain injury between the two groups. There were 465 and 1,554 patients who experienced traumatic brain injury with and without sleep apnea, respectively. Among them, 6 and 10 patients died of this traumatic brain injury in the cohorts with and without sleep apnea, respectively. The difference in mortality for traumatic brain injury patients was not statistically significant between the two groups.

Compared with individuals in the cohort without sleep apnea, sleep apnea patients with CPAP had a much higher risk of developing head injury (adjusted HR 1.79, 95\% CI, 1.27-2.53) (Table 5).

Table 6 presents the risk of head injury for different drug exposure durations (cumulative exposure day). Compared with the non-BZD cohort, patients who had received benzodiazepine for $<10,10-50,50-240$, and $>240$ days exhibited substantially decreased risks of head injury.

\section{Discussion}

Our study showed that sleep apnea, whether coupled with sedative or psychiatric medications, was associated with an increased risk of traumatic brain injury. Furthermore, sleep apnea patients aged 65-79 years had the highest risk of traumatic brain injury. It has been previously reported that use of sedative and hypnotic medications was associated with falls, especially in elderly patients (21-23). Avoiding sedatives use in those with high risk of falls appeared to be a protective strategy in decreasing events of accidental falling. In 2015, Jennings and colleagues published a simple screening item to identify high fall-risk groups in hospital settings (24). However, we discovered that sleep apnea was also associated with a higher risk of traumatic brain injury. The mechanism underlying the association between sleep apnea and traumatic brain injury remains to be investigated. Although information regarding etiologies involved in traumatic brain injuries were unavailable in the NHIRD, we proposed several possible explanations for 
Table 5 Comparisons of incidence and hazard ratios of head injury by treatment of sleep disorders

\begin{tabular}{lcccccc}
\hline Variables & $\mathrm{N}$ & Event & $\mathrm{IR}$ & Crude HR $(95 \% \mathrm{Cl})$ & Adjusted HR (95\% Cl) & $\mathrm{P}$ value \\
\hline Control & 25,824 & 1,554 & 10.5 & 1 (Reference) & 1 (Reference) \\
Sleep apnea & & & & & & \\
Without CPAP & 6,153 & 431 & 12.2 & $1.17(1.05-1.30)^{\star \star}$ & $1.20(1.08-1.34)$ \\
With CPAP & 303 & 34 & 22.7 & $2.15(1.53-3.03)^{\star * *}$ & $1.79(1.27-2.53)$ & 0.001 \\
\hline
\end{tabular}

$\mathrm{IR}$, incidence rate, per 1,000 person-years; HR, hazard ratio; $\mathrm{Cl}$, confidence interval. Variables found to be statistically significant in the univariable model were further included in the multivariable model. Adjusted for sex, age, diabetes, hypertension, spinal cord injury, osteoporosis, fracture, alcohol-related disease, cirrhosis, cataract, macular degeneration, stroke, CKD or ESRD, dementia, and benzodiazepine, non-benzodiazepine, and antipsychotic medication use. ${ }^{\star *}, \mathrm{P}<0.01$; ${ }^{\star \star \star}, \mathrm{P}<0.001$.

Table 6 Incidence and adjusted hazard ratios of head injury stratified by duration of benzodiazepine and non-benzodiazepine use in study cohort

\begin{tabular}{|c|c|c|c|c|c|}
\hline Medication & $\mathrm{N}$ & Event & Person-year & $\mathrm{IR}$ & Adjusted HR (95\% Cl) \\
\hline \multicolumn{6}{|l|}{ Benzodiazepine } \\
\hline$<10$ days & 2,112 & 97 & 15,062 & 6.44 & $0.56(0.45-0.69)^{\star \star \star}$ \\
\hline 10-50 days & 3,691 & 213 & 25,451 & 8.37 & $0.66(0.57-0.77)^{\star \star \star}$ \\
\hline$>240$ days & 5,426 & 451 & 33,327 & 13.5 & $0.77(0.68-0.88)^{\star \star \star}$ \\
\hline Without non-BZD & 26,112 & 1,481 & 144,661 & 10.2 & 1.00 \\
\hline \multicolumn{6}{|l|}{ Non-BZD } \\
\hline$<10$ days & 1,075 & 76 & 7,409 & 10.3 & $0.90(0.72-1.14)$ \\
\hline
\end{tabular}

$\mathrm{IR}$, incidence rate, per 1,000 person-years; $\mathrm{HR}$, hazard ratio; $\mathrm{Cl}$, confidence interval. Variables found to be statistically significant in the univariable model were further included in the multivariable model. Adjusted for sex, age, diabetes, hypertension, spinal cord injury, osteoporosis, fracture, alcohol-related disease, cirrhosis, cataract, macular degeneration, stroke, CKD or ESRD, dementia, and benzodiazepine, non-benzodiazepine, and antipsychotic medication use. ${ }^{* \star}, \mathrm{P}<0.001$.

this finding, as the main causes of traumatic brain injuries include falls and traffic accidents. First, sleep apnea is reportedly prevalent among several movement disorders, such as Parkinson's disease (25). Parkinson's disease and associated movement disorders can cause imbalance of muscle tone and regulation, which could confer a high risk of falls. Second, sleep disorders, of which insomnia and sleep apnea make up a majority of cases, can cause poor attention and concentration while leading to more accidents in daily activities $(26,27)$. Third, sleep apnea patients might have more medical visits, tend to seek medical service while injured, and have more chances to get the diagnosis of traumatic brain injuries compared with patients without sleep disorders.

In contrast to previous studies that focused on the increased risk of sleep disorders in people who experienced traumatic brain injuries (24-26), we found that sleep apnea patients had a higher risk of traumatic brain injury. Furthermore, we found that irrespective of hypnotic use status, the risk of traumatic brain injuries was nonsignificant in sleep disorder patients. In our study, sleep apnea patients did not have a higher mortality and more ICU hospitalization because of traumatic brain injuries compared with patients without sleep disorders. Additionally, it is 
noteworthy that sleep apnea patients with CPAP use had a higher risk compared with sleep apnea patients without CPAP use. Karimi et al. reported that CPAP use reduced the risk of motor vehicle accidents, but not the risk of brain injuries (27). Therefore, we propose that CPAP use could be an index for severity of sleep apnea, and patients requiring CPAP might be more vulnerable to brain injuries because of previous long-term nighttime deoxygenating.

Our data may imply an indirect relationship between brain injury and sleep apnea (e.g., through a fall or a driving accident). Notably, these traumatic brain injuries can be preventable in sleep apnea patients if sleep apnea is cured. Because brain injuries are associated with increased medical costs and increased risk of dementia (26), attempting to cure sleep apnea rather than focusing on treating or alleviating its symptoms is clinically valuable.

Burks et al. reported that nonadherence with employermandated sleep apnea treatment could be associated with increased risk of serious truck crashes (28). Therefore, alternative strategy, stimulus control, progressive muscle relaxation, paradoxical intention sleep restriction, biofeedback, multifaceted cognitive-behavior therapy for insomnia (29) as well as weight loss, dental devices, and CPAP for sleep apnea might have benefits in reducing the incidence of sleep disorders and associated complications. Additionally, severe sleep apnea patients might need surgical intervention. Further studies are warranted to examine these nonpharmacological treatments in decreasing events of traumatic brain injuries in sleep disorder patients (30).

Several limitations of this study should be mentioned. First, information about personal habits of alcohol consumption, daily sun exposure, daily caffeine intake, vision accuracy, safety of environment, body mass index, obesity, and daily function ability are factors possibly associated with traumatic brain injuries and sleep quality that were unavailable in the NHIRD. We also had no information regarding apnea/hypopnea index, which can be used to classify sleep apnea severity. Therefore, whether the CPAP group differed from the non-CPAP group on severity of sleep apnea was not known. The information regarding etiologies of traumatic brain injuries that are outside of a person's control, such as assault, pedestrian being struck by a motor vehicle, being struck in the head by an external object were lacking. Therefore, some etiologies may have less to do with cognitive dysfunction resulting from a sleep disorder. Second, we did not adjust medical visits in our study; thus, sleep apnea patients might have had more chances to receive a diagnosis of traumatic brain injury compared with patients without sleep apnea. However, because we recorded these based on emergency room database information, this bias should be minimized. Third, detailed imaging reports and medical records regarding traumatic wounds were also unavailable in the NHIRD. Therefore, we could not classify the severity of traumatic brain injuries. Fourth, CPAP use compliance was not obtainable from the NHIRD, and whether the patients had used concomitant treatment strategies, including losing weight, decreasing alcohol consumption, or quitting smoking were unknown. Finally, the diagnostic rate of obstructive sleep apnea is quite low. Thus, there would be a certain percentage of patients identified as not having sleep apnea who actually had sleep apnea, although undiagnosed at the time. Therefore, the increased risks of traumatic brain injuries in sleep apnea patients compared with patients without sleep apnea would be underestimated in our study and further strengthen our findings that sleep apnea patients had increased risk of traumatic brain injuries. In conclusion, sleep apnea patients are at a higher risk of traumatic brain injuries. Although our data showed an association between CPAP use and higher risk of brain injuries, the causative relationship was not definitively established because of the limitations of this study. Further studies are needed to evaluate the effects of CPAP on incidences of traumatic brain injuries in sleep apnea patients, especially in elderly patients.

\section{Acknowledgments}

Funding: This work was supported by grants from the Ministry of Health and Welfare, Taiwan (MOHW108TDU-B-212-133004), China Medical University Hospital (CMU106-ASIA-12, DMR-107-192); Academia Sinica Stroke Biosignature Project (BM10701010021); MOST Clinical Trial Consortium for Stroke (MOST 107-2321-B039-004); Tseng-Lien Lin Foundation, Taichung, Taiwan; and Katsuzo and Kiyo Aoshima Memorial Funds, Japan. The funders had no role in study design, data collection and analysis, decision to publish, or preparation of the manuscript. No additional external funding received for this study.

\section{Footnote}

Conflicts of Interest: The authors have no conflicts of interest 
to declare.

Ethical Statement: The authors are accountable for all aspects of the work in ensuring that questions related to the accuracy or integrity of any part of the work are appropriately investigated and resolved. This study was approved as fulfilling the conditions for exemption by the Institutional Review Board (IRB) of China Medical University (CMUH104-REC2-115-CR4). The IRB also specifically waived the consent requirement.

\section{References}

1. Jean-Louis G, Zizi F, Clark LT, et al. Obstructive sleep apnea and cardiovascular disease: role of the metabolic syndrome and its components. J Clin Sleep Med 2008;4:261-72.

2 Yaggi HK, Concato J, Kernan WN, et al. Obstructive sleep apnea as a risk factor for stroke and death. $\mathrm{N} \mathrm{Engl} \mathrm{J}$ Med 2005;353:2034-41.

3 Postuma RB, Gagnon JF, Vendette M, et al. Quantifying the risk of neurodegenerative disease in idiopathic REM sleep behavior disorder. Neurology 2009;72:1296-300.

4 Vgontzas AN, Liao D, Pejovic S, et al. Insomnia with objective short sleep duration is associated with type 2 diabetes: A population-based study. Diabetes Care 2009;32:1980-5.

5 Liu J, Hay J, Faught BE. The Association of Sleep Disorder, Obesity Status, and Diabetes Mellitus among US Adults-The NHANES 2009-2010 Survey Results. Int J Endocrinol 2013;2013:234129.

6 Lavie L. Oxidative stress in obstructive sleep apnea and intermittent hypoxia--revisited--the bad ugly and good: implications to the heart and brain. Sleep Med Rev 2015;20:27-45.

7 Li L, Zhang X, Yang D, et al. Hypoxia increases Abeta generation by altering beta- and gamma-cleavage of APP. Neurobiol Aging 2009;30:1091-8.

8 Da Luz P, Libby P, Laurindo F, et al. Endothelium and Cardiovascular Diseases. 1st Edition. Vascular Biology and Clinical Syndromes. 19th January 2018. eBook ISBN: 9780128125519.

9 Hargens TA, Kaleth AS, Edwards ES, et al. Association between sleep disorders, obesity, and exercise: a review. Nat Sci Sleep 2013;5:27-35.

10 Lavie L, Perelman A, Lavie P. Plasma homocysteine levels in obstructive sleep apnea: association with cardiovascular morbidity. Chest 2001;120:900-8.
11 Mazza S, Pépin JL, Naëgelé B, et al. Most obstructive sleep apnoea patients exhibit vigilance and attention deficits on an extended battery of tests. Eur Respir J 2005;25:75-80.

12 Surman CB, Adamson JJ, Petty C, et al. Association between attention-deficit/hyperactivity disorder and sleep impairment in adulthood: evidence from a large controlled study. J Clin Psychiatry 2009;70:1523-9.

13 Database NHIR. Taiwan, Available online: http://nhird. nhri.org.tw/en/index.html

14 Shen TC, Lin CL, Wei CC, et al. Risk of Obstructive Sleep Apnea in Adult Patients with Asthma: A Population-Based Cohort Study in Taiwan. PLoS One 2015;10:e0128461.

15 Wang SH, Chen WS, Tang SE, et al. Benzodiazepines Associated With Acute Respiratory Failure in Patients With Obstructive Sleep Apnea. Front Pharmacol 2019;9:1513.

16 Lee YC, Hung SY, Wang HK, et al. Sleep apnea and the risk of chronic kidney disease: a nationwide populationbased cohort study. Sleep 2015;38:213-21.

17 Harnod T, Wang YC, Kao CH. Association Between Benzodiazepine Use and Epilepsy Occurrence: A Nationwide Population-Based Case-Control Study. Medicine (Baltimore) 2015 ;94:e1571.

18 Huang WS, Muo CH, Chang SN, et al. Benzodiazepine use and risk of stroke: a retrospective population-based cohort study. Psychiatry Clin Neurosci 2014;68:255-62.

19 Wu CS, Tsai YT, Tsai HJ. Antipsychotic drugs and the risk of ventricular arrhythmia and/or sudden cardiac death: a nation-wide case-crossover study. J Am Heart Assoc 2015;4. doi: 10.1161/JAHA.114.001568.

20 Egea-Guerrero JJ, Rodríguez-Rodríguez A, GordilloEscobar E, et al. IMPACT Score for Traumatic Brain Injury: Validation of the Prognostic Tool in a Spanish Cohort. J Head Trauma Rehabil 2018;33:46-52.

21 Mendelson WB. The use of sedative/hypnotic medication and its correlation with falling down in the hospital. Sleep 1996;19:698-701.

22 Sorock GS, Shimkin EE. Benzodiazepine sedatives and the risk of falling in a community-dwelling elderly cohort. Arch Intern Med 1988;148:2441-4.

23 Riefkohl EZ, Bieber HL, Burlingame MB, et al. Medications and falls in the elderly: a review of the evidence and practical considerations. $\mathrm{P}$ and $\mathrm{T}$ 2003;28:724-33.

24 Jennings LA, Reuben DB, Kim SB, et al. Targeting a highrisk group for fall prevention: strategies for health plans. 
Am J Manag Care. 2015;21:e519-26.

25 Tan A, Salgado M, Fahn S. Rapid eye movement sleep behavior disorder preceding Parkinson's disease with therapeutic response to levodopa. Mov Disord 1996;11:214-6.

26 Shively S, Scher AI, Perl DP, et al. Dementia resulting from traumatic brain injury: what is the pathology? Arch Neurol 2012;69:1245-51.

27 Karimi M, Hedner J, Häbel H, et al. Sleep apnea related risk of motor vehicle accidents is reduced by continuous positive airway pressure: Swedish traffic accident registry data. Sleep 2015;38:341-9.

Cite this article as: Lin SY, Chen W, Harnod T, Lin CL, Hsu WH, Lin CC, Chang YL, Wang IK, Kao CH. Sleep apnea and risk of traumatic brain injury and associated mortality and healthcare costs: a population-based cohort study. Ann Transl Med 2019;7(22):644. doi: 10.21037/atm.2019.10.88
28 Burks SV, Anderson JE, Bombyk M, et al. Nonadherence with Employer-Mandated Sleep Apnea Treatment and Increased Risk of Serious Truck Crashes. Sleep 2016;39:967-75.

29 Morin CM, Hauri PJ, Espie CA, et al. Nonpharmacologic treatment of chronic insomnia. An American Academy of Sleep Medicine review. Sleep 1999;22:1134-56.

30 Jullian-Desayes I, Joyeux-Faure M, Tamisier R, et al. Impact of obstructive sleep apnea treatment by continuous positive airway pressure on cardiometabolic biomarkers: a systematic review from sham CPAP randomized controlled trials. Sleep Med Rev 2015;21:23-38. 